How to reference this article

Giacobbe Borelli, M. (2019). Attrici d'avanguardia e teatro femminista a Roma negli anni Settanta: nuovi luoghi e linguaggi. Italica Wratislaviensia, 10(2), 257-273.

DOI: http://dx.doi.org/10.15804/IW.2019.10.1.28

Maia Giacobbe Borelli

Progetto ORMETE, Istituto centrale per i Beni sonori e audiovisivi di Roma e Museo Biblioteca dell'Attore di Genova

2maiaborelli@gmail.com

\title{
ATTRICI D'AVANGUARDIA E TEATRO FEMMINISTA A ROMA NEGLI ANNI SETTANTA: NUOVI LUOGHI E LINGUAGGI
}

\author{
AVANT-GARDE ACTRESSES AND FEMINIST \\ THEATRE IN ROME IN THE 1970s: NEW STAGES \\ AND NEW FORMS OF EXPRESSION
}

\begin{abstract}
This essay is based on one of the ORMETE (Oralità Memoria Teatro: www.ormete.net) projects: Donne di teatro a Roma ai tempi della mobilitazione femminista (1965-1985). The research shows the inter-relations between the feminist movement and the actresses of the avant-garde scene in Rome.

The focus of each of the women's personal narrations is characterised by its strong and definite feminist or anti-conformist identity and by its determination to channel its own imagery in the experimental scene of the avant-garde. Many of the women gathered in feminist companies or joined collective groups; some ventured the road of dramaturgy, while still others dared more alternative, experimental kinds of solo performances. This text sheds light on a variety of alternative spaces for performances in Rome. It is there where these new forms of theatrical expressions were born.
\end{abstract}

Keywords: avant-garde theatre, feminism, New Theatre, Rome, 1974-1979 
Cliente: Mica le donne fanno così. Manila: E come fanno? Cliente: Un po' più di civetteria, di garbo, che ne so...scodinzolano, fanno le moine. Manila: Io non scodinzolo perché non sono un cane. Spogliati!

(Maraini, 2001, p. 9)

egli anni caldi della mobilitazione femminista Dacia Maraini de-
nunciava su "effe" l'immobilismo del teatro di tradizione rispetto alla questione femminile:

Le donne stanno prendendo coscienza, sempre più numerose in tutto il paese. E cosa succede nel mondo dello spettacolo? Poco o niente che rifletta questa presa di coscienza. Il fatto è che alla diffusione del femminismo non ha corrisposto una presa di potere, anche minima, da parte delle donne. Il potere (di esprimersi, di fare progetti, di organizzare spettacoli, di ottenere sussidi) è ancora saldamente in mano agli uomini, registi, amministratori, impresari che siano. Questa è la ragione per cui, mentre tutto sta cambiando nella testa e nel cuore delle donne, sul palcoscenico si continuano a recitare vecchi drammi di sopraffazione, di sadismo, di violenza sulla donna. (1976, p. 24)

Se gli ambienti del teatro di tradizione sembravano impermeabili al protagonismo delle teatranti, Dacia Maraini e le compagne di strada, che vivevano in prima persona quella calda stagione di mobilitazione, risposero creando palcoscenici, compagnie, spazi e registri espressivi che misero al centro la soggettività e l'empowerment del genere femminile.

Il mio contributo si concentra su quanto accadde a Roma, fra il teatro di ricerca e l'attivismo femminista, in un ristretto giro d'anni, fra il 1974 e il 1979, parendomi quelli che registrano la massima vitalità delle scene militanti ${ }^{1}$. Punto di partenza di questa cronologia è l'approdo de La donna perfetta, una pièce di Dacia Maraini sull'aborto, alla Biennale di teatro di Venezia, appunto nel 1974; punto di provvisoria

1 Ringrazio Roberta Gandolfi per il confronto e la discussione prestata in fase di stesura del presente contributo. 
chiusura del mio discorso è la ricchezza dei debutti ascrivibili alla scena romana delle donne nel 1979: un nuovo spettacolo di Maraini, Suor Juana, il 6 dicembre 1979, ma anche spettacoli che divennero subito cult quali Molly Cara, di e con Piera degli Esposti; la performance Mine $\mathrm{Ha}$, l'educazione delle ragazze, che Rossella Or dirige utilizzando l'originale spazio della Piscina del CONI, mentre quell'anno Lucia Poli scrive, interpreta e mette in scena il monologo Liquidi al Teatro Alberichino, spazio che aveva già utilizzato due anni prima Marilù Prati per raccontare con Bambulé il suo percorso esistenziale.

Punto culminante, nel novembre 1979 al Teatro La Maddalena, è la rassegna La scimmia viola, che riunisce in uno spazio femminista tante autrici-attrici, icone dell'avanguardia romana. Tra queste Or, Poli e Vasilicò ${ }^{2}$. E intanto nascono a Roma compagnie di giovanissime, quali il Teatro Viola (1979-1983) ${ }^{3}$ e TheATre $(1978-1980)^{4}$.

Il periodo così circoscritto coincide, non a caso, con la fase di massima mobilitazione del movimento delle donne, con le grandi manifestazioni di piazza, di un gioioso e affollatissimo 8 marzo a Piazza Farnese nel 1976 (Ill. 1), e della conquista di uno spazio nuovo, quella Casa delle Donne, sita all'interno di un grande palazzo abbandonato dal Comune che viene occupato in quello stesso anno e che resterà per alcuni anni il principale luogo di dibattito, incontri e spettacoli del movimento femminista ${ }^{5}$.

2 Rassegna La scimmia viola organizzata da Carmen Pignataro con: R. Or, L. Poli, L. Vasilicò, Teatro Viola, Gruppo Isabella Morra, G. Mainardi, T. Gatta, P. Molero.

3 Teatro Viola, di e con F. D’Andreamatteo, M. Giacobbe Borelli, E. Mereghetti. Cfr. Intervista a Teatro Viola di Francesca Fava, Roma 14 febbraio 2015. Tra le recensioni su riviste americane, segnaliamo Jill Dolan sulle pagine di "The Drama Review" (Dolan, 1982 e Fava, 2015).

4 TheATre, formato dalle performer Gloria Guasti, Ippolita Avalli, Marzia Mealli e Caterina Casini, apprezzato da Achille Bonito Oliva, Mario Schifano e Giuseppe Bartolucci, partecipò agli incontri di Pistoia e di Caserta, ma anche nel 1978 all'occupazione della Casa delle Donne.

5 L'occupazione della Casa delle Donne di via del Governo Vecchio finirà nel 1984 quando il Comune di Roma offrirà un altro luogo, il Buon Pastore, sito in via della Lungara e ristrutturato appositamente, come sede permanente delle molteplici attività del movimento. 


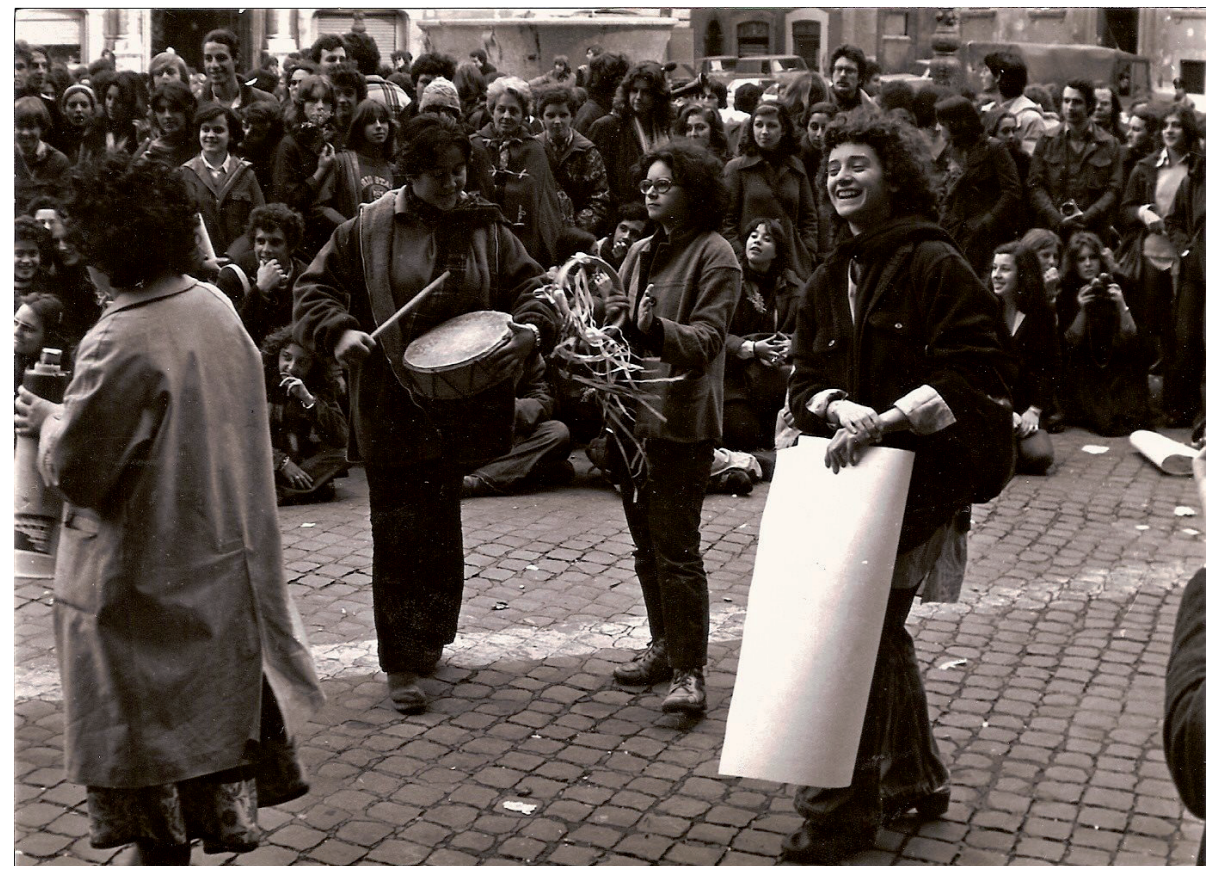

ILL. 1. Foto della manifestazione femminista a piazza Farnese l'8 marzo 1976

Importanti innovazioni sociali e politiche percorrevano l'Italia di quegli anni e Roma in particolare, in quanto sua capitale: dal referendum sul divorzio del maggio 1974 alla riforma del diritto di famiglia del 1975, l'esplosione della creatività del movimento studentesco nel '77, dalla legge del 1978 che regola l'interruzione di gravidanza ${ }^{6}$, frutto di una lunga mobilitazione iniziata il 6 dicembre 1975 con la prima grande manifestazione nazionale per la depenalizzazione dell' aborto $^{7}$, alla costituzione dei collettivi femministi che mirano alla realizzazione della sorellanza come strumento per rafforzare i percorsi individuali. Tra questi: il Collettivo San Lorenzo di Simonetta Tosi che pratica il self-help,

6 L. 194/78 del 22 maggio 1978.

7 Manifestazione nazionale a Roma a cura del CRAC, la prima ad essere interamente femminile, convocata per la depenalizzazione dell'aborto, ebbe 20.000 donne partecipanti. 
i gruppi di autocoscienza del collettivo femminista-comunista di via Pomponazzi, il Movimento Femminista Romano di via Pompeo Magno, il Movimento di Liberazione della Donna e i Collettivi Donne di tanti quartieri di Roma ${ }^{8}$.

Lo spettacolo Molly cara $^{9}$, monologo con Piera Degli Esposti e la regia di Ida Bassignano, tratto da Joyce, segnerà tutto il 1979, dal debutto a Milano al Teatro Uomo il 25 gennaio, alla tournée in tutta Italia nei mesi successivi. Di questo spettacolo, Premio Ubu della stagione 1978-79, e primo della lunga stagione di successi dell'attrice, ha testimoniato per noi sia la regista Bassignano (Bassignano, 2016) ${ }^{10}$, che Sista Bramini, regista e attrice, in quegli anni giovane spettatrice, che considera centrale per le sue future scelte professionali proprio esserne stata spettatrice nel 1979 (Bramini, 2015) ${ }^{11}$.

Piera Degli Esposti sperimenta con la voce un registro espressivo originale per rendere il flusso interiore di coscienza di una donna che

8 Per la storia del movimento femminista romano cfr. almeno Stelliferi, 2015 e Lussana, 2012.

${ }^{9}$ Un estratto di questo spettacolo è disponibile su Youtube a https://www.youtube. $\mathrm{com} /$ watch? $=$ =IcUR31SoVfQ.

${ }^{10}$ Il monologo di Molly Bloom, cioè l'ultimo capitolo dell'Ulisse di James Joyce (pubblicato per la prima volta nel ' 22 e tradotto in Italia nel '60) divenne in quegli anni uno spettacolo cult con il titolo Molly cara. Dall'intervista alla regista Ida Bassignano per il Progetto ORMETE: «la novità dell'impresa (un'ora e un quarto d'ininterrotto flusso di coscienza), l'autorevolezza dell'autore, la straordinaria prova dell'attrice ne fecero da subito un successo. [...] Ricordo di aver dovuto spingere in scena una Piera terrorizzata, ricordo il silenzio ingessato del pubblico milanese per i primi trenta minuti di spettacolo: eppure la prova che l'emozione montava fu l'esplosione in una risata liberatoria ad una frase blandamente comica ("Mi sa che in Cina a quest'ora si pettinano i codini per la giornata"), quasi si fossero infine persuasi che si poteva godere 'fisicamente' lo spettacolo, come poi avvenne in un crescendo continuo fino al finale. Il trasferimento dal letterario monologo interiore alla fisicità teatrale era avvenuto principalmente attraverso la voce, la voce di Piera Degli Esposti, che fu definita 'vocestrumento': una voce spezzata e dai molti toni, che mimava o accennava ai movimenti dell'inconscio attraverso una resa fonica quasi cantata, capace di far diventare il materiale letterario una sorta di 'ballata', come fu scritto» (Bassignano, 2016).

11 Sista Bramini nel 1986 fonderà TeatroNatura, compagnia a prevalenza femminile, ancora attiva in una fase post-femminista come quella contemporanea. 
s'interroga sulla sessualità maschile, in modo diretto e ironico come mai si era sentito prima.

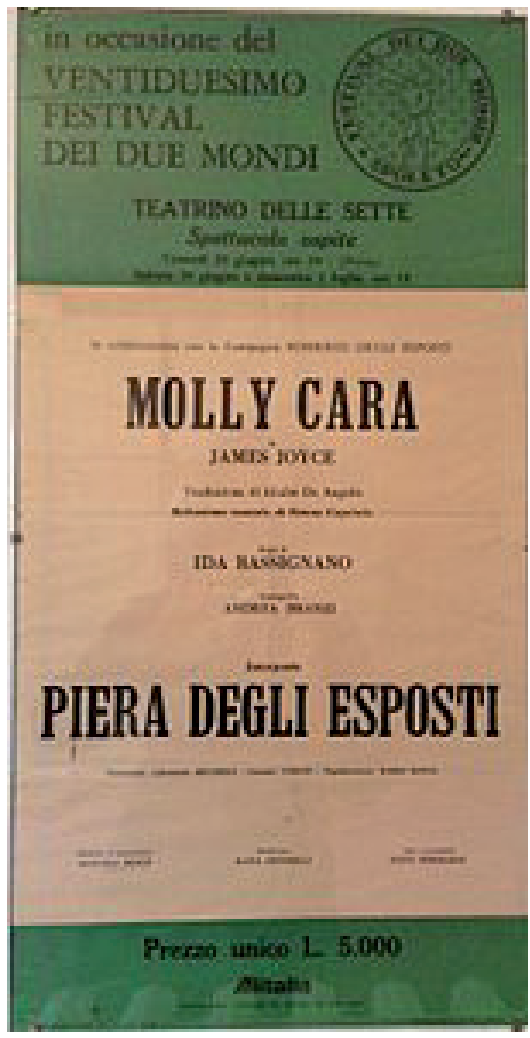

ILL. 3. Foto della locandina di Molly cara al Festival dei Due Mondi di Spoleto nel 1979

La Storia di Elsa Morante è il romanzo che apre il periodo che abbiamo preso in esame; Goffredo Fofi ne parlerà come di «un grande affresco materno» (Fofi, 1979, p. 98). L'opera, protagonista Ida, una giovane vedova che alleva da sola due figli, esce in edizione economica nel giugno $1974^{12}$.

12 In pochi mesi La Storia (Torino: Einaudi) raggiungerà le 600.000 copie vendute, grazie anche al prezzo politico di 2.000 lire imposto dalla scrittrice, nonostante le critiche prevalentemente negative provenienti dal mondo letterario più in vista, in maggio- 
La figura di Elsa Morante (Roma 1912-Roma 1985), è citata come esempio nelle interviste che abbiamo realizzato da chi non era associata al movimento femminista, come Marilù Prati, che pur aveva vissuto sulla propria pelle le difficoltà di conciliare la condizione professionale con la maternità (Prati, 2015). Una letterata come la Morante, non legata al movimento di lotta femminista, diviene, suo malgrado, punto di riferimento per le artiste che sono alla ricerca di una propria autonomia. Così come sarà per Pier Paolo Pasolini, spettatore anticonformista dei teatri d'avanguardia e narratore acuto degli eventi che precedono gli anni in questione, delle novità e contraddizioni insite nei processi culturali in corso, assassinato a Ostia, periferia di Roma, il 2 novembre 1975. La sua morte scuote profondamente il mondo romano, compreso chi frequenta gli ambienti teatrali dell'avanguardia.

Non va trascurato il clima pesante imposto dalle stragi e dagli attentati nelle piazze in tutta Italia ${ }^{13}$, che culmina nel 1978 a Roma col rapimento e l'uccisione di Aldo Moro $^{14}$, grave prova per la nostra democrazia.

È dunque in mezzo a tutte queste diverse spinte e sollecitazioni, che si è formata la teatralità specifica che le attrici fecero emergere negli ambienti teatrali romani di quegli anni. Alcuni elementi della lotta politica delle donne sono entrati in modo del tutto naturale nella narrazione di sé delle professioniste operanti in quegli anni a Roma. E dai luoghi teatrali, in modo speculare, emergono risonanze che li colorano di contenuti legati alla rivendicazione di una nuova espressività femminile. Le attrici cominciano a far sentire la loro voce scrivendo testi e mettendosi

ranza maschile, da Cases ad Asor Rosa a Barilli, da Pasolini a Luperini, da Balestrini a Siciliano, e il dibattito scatenato sul quotidiano "Il Manifesto", sulle riviste "Quaderni Piacentini", "Belfagor", "Ombre rosse" e altre.

13 La prima strage degli anni di piombo italiani è quella di Piazza Fontana a Milano, 12 dicembre 1969, con 17 morti; seguirà la strage di Piazza della Loggia a Brescia il 28 maggio 1974, che provocò 8 morti e 102 feriti durante un comizio antifascista e il 4 agosto 1974 l'attentato dell'Italicus, con 12 morti. Poi sarà la volta della strage alla stazione di Bologna del 2 agosto 1980 che causerà ben 85 morti.

14 Aldo Moro fu sequestrato dai terroristi delle Brigate Rosse in via Fani il 16 marzo 1978 e ritrovato morto il 9 maggio. 
in scena, spinte dagli eventi a riprendersi la parola. Oltre agli spazi femministi, i luoghi dell'avanguardia si rendono disponibili a ospitarle.

\section{LE RADICI: LA RICERCA STORICA, LE TESTIMONIANZE ORALI}

Ciò che mi permette di aprire il discorso sull'intreccio fra la mobilitazione femminista e le teatranti romane, un territorio davvero poco studiato e documentato, è una ricerca in corso di svolgimento, Donne di teatro a Roma ai tempi della mobilitazione femminista (1965-1985), che porto avanti con Francesca Fava e Roberta Gandolfi, con l'obiettivo appunto di documentare, attraverso una serie d'interviste audio con le protagoniste dell'epoca, la varietà degli intrecci fra i percorsi delle teatranti romane e il movimento femminista ${ }^{15}$.

Al momento abbiamo raccolto le testimonianze orali di ventuno protagoniste della scena romana fra gli anni Sessanta e gli anni Ottanta: si tratta principalmente di attrici, ma anche autrici, registe, scenografe e organizzatrici. Ci siamo rivolte in prima battuta a chi condivideva e sosteneva gli orizzonti e le pratiche dei gruppi legati alle rivendicazioni femministe, per poi sondare gli ambienti delle compagnie teatrali neo-avanguardiste e le figure di chi agiva autonomamente sulle scene dello spettacolo. Le ventuno testimoni attraversano, con le loro biografie professionali, la scrittura, la messinscena, l'impresariato, oltre che, ovviamente, la dimensione attoriale a Roma ${ }^{16}$. Oltre alle attrici del femminismo militante (Saviana Scalfi e Renata Zamengo principalmente)

15 La ricerca si svolge nell'ambito del Progetto ORMETE, acronimo di Oralità Memoria Teatro, un progetto molto articolato, diretto da Donatella Orecchia e Livia Cavaglieri, che costruisce e raccoglie testimonianze orali riguardanti il teatro italiano della seconda metà del XX secolo; è svolta in collaborazione con l'Istituto centrale per i Beni sonori e audiovisivi di Roma e il Museo Biblioteca dell'Attore di Genova, depositari dell'archivio fisico delle fonti sonore raccolte e aperte alla consultazione degli studiosi. Vedi: http://patrimoniorale.ormete.net e http:/www.ormete.net.

${ }^{16}$ Cfr. il link: http://patrimoniorale.ormete.net/progetto/donne-di-teatro-a-roma-aitempi-della-mobilitazione-femminista-1965-1985. Su questo sito stiamo completando i metadati delle testimonianze raccolte per implementare la documentazione on-line. 
abbiamo intervistato attrici-icona delle cantine romane, che prestavano viso e corpo alle scritture sceniche dei loro ben più noti compagni, quali Lydia Mancinelli, che fu per tanti anni l'organizzatrice, la compagna e la prima attrice di Carmelo Bene, ma anche le protagoniste degli spettacoli più noti delle cantine romane, come Rossella Or (Ill. 2), che debutta nel 1973 con Pirandello chi? di Memé Perlini, e recita poi con Simone Carella e Mario Prosperi (Or, 2015) ${ }^{17}$, Lucia Vasilicò, presente in tanti

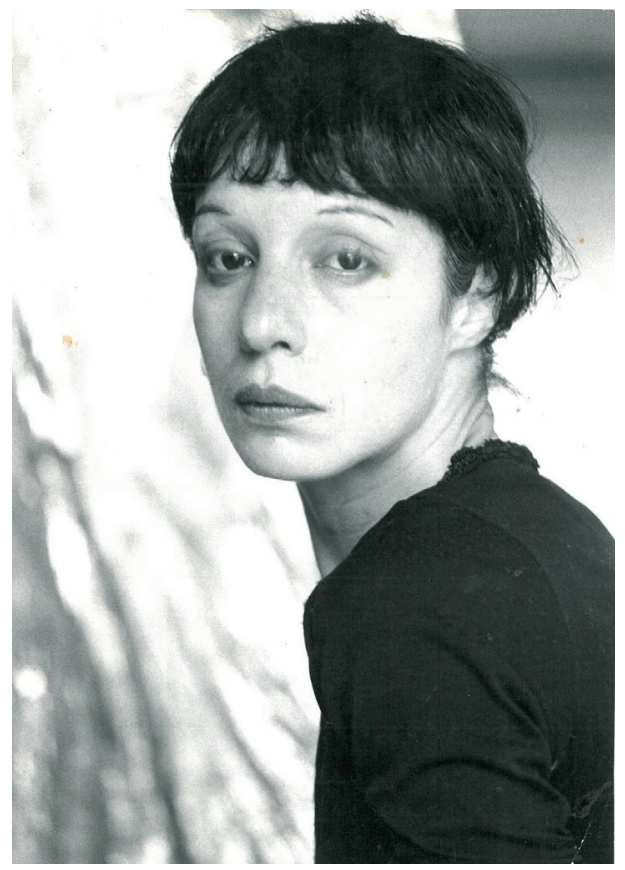

ILL. 2. Foto dell’attrice Rossella Or

17 Or è attrice di Memé Perlini e Simone Carella dal 1973 al 1979, con Mario Prosperi dal 1980 al 1989. Debutta come autrice e regista di se stessa nella performance Corpovoce, Solo performance del 1977, dove è ancora "corpo senza parola", a cui segue Respiro sospeso, monologo del 1978 prodotto dal Beat72, dove finalmente "riacquista la parola". Seguirà un altro monologo, Non come loro da lei scritto e interpretato; l'esperienza di Mine Ha (1979); Oh (1982) per l'apertura dello spazio dell'Uccelliera di Villa Borghese; Lei (1993-1996) dove coniuga performance e teatro di parola, sulla violenza contro le donne durante la guerra in Jugoslavia, la partecipazione al film rievocativo "Estate Romana" (1999) di Matteo Garrone, in cui recita se stessa. 
spettacoli del più noto fratello, o Marilù Prati, che incontra l'esperienza del Granteatro di Carlo Cecchi per poi lavorare con Eduardo De Filippo. E un'attrice-autrice cresciuta all'ombra del fratello, Lucia Poli, che ha poi debuttato da sola con Liquidi, monologo dove mette in scena gli umori, solitamente nascosti, che segnano il corpo della donna, dal latte al mestruo (Poli, 2014), o come l'attrice-manager Manuela Morosini, che ha creato il teatro Spaziozero, entrambe con proprie compagnie professionali e un rapporto ambivalente con la mobilitazione femminista.

Chi ha invece tenuto strettamente legate le due parti, nuovo teatro e movimento femminista, è stato Teatro Viola, riportando nelle piazze femministe quanto appreso nei seminari del teatro di gruppo, per declinarlo al femminile. Questo gruppo di ragazze, poco noto in Italia se non negli ambienti del femminismo romano o per alcune recensioni di Nico Garrone sul quotidiano "La Repubblica", ha partecipato a Copenaghen e New York a festival internazionali di teatro delle donne, dove è apprezzato per il suo registro comico e surreale.

I metodi della storia orale, che da sempre è storia dal basso che restituisce memoria degli eventi e dei fenomeni storici marginalizzati dal canone storiografico, sono parsi i migliori per la ricostruzione di un quadro d'insieme che ai nostri occhi si presenta complesso e sfaccettato.

Il lavoro di raccolta delle fonti orali che stiamo compiendo non è solo la riscoperta dei luoghi, delle produzioni artistiche, delle compagnie, ma anche un'immersione nel mondo delicato delle sensibilità individuali, un'indagine nelle memorie, per rievocare le atmosfere all'interno delle quali si sono rinnovate in modo radicale sia le relazioni professionali che quelle private delle attrici, delle drammaturghe, registe e organizzatrici della scena romana. Obiettivo primo del nostro progetto è dunque contribuire alla memoria storica, tenendo conto del fatto che questa del punto di vista femminile è una storia assente dalla letteratura critica del nuovo teatro a Roma, anche dai tanti volumi e studi che sono usciti negli ultimi anni ${ }^{18}$.

Attingendo dunque al mare aperto di questa ricerca, impossibile a circoscriversi in un saggio, intendo proporre un doppio squarcio sul

${ }^{18}$ Cfr. bibliografia. 
periodo 1974-1979 che dischiuda una sorta di mobile mappa per luoghi, per interrogare le intersezioni fra i luoghi del movimento delle donne e i luoghi delle teatranti; e proporre un affondo sulla polifonia dei registri espressivi che caratterizzava le scene delle donne.

\section{I LUOGHI DEL MOVIMENTO, I LUOGHI DELLE TEATRANTI}

Dacia Maraini è il punto di riferimento della cultura femminista di quegli anni, e con lei abbiamo terminato le nostre interviste. La sua battaglia personale è stata quella di ridare la parola a tutte le donne, anche nel mondo teatrale istituzionale, per restituire loro dignità offrendo non solo testi, tradotti in tutto il mondo, ma anche un luogo, il Teatro la Maddalena, aperto alla fine del 1973 e chiuso nel 1989, che diviene il luogo principale della riflessione culturale del movimento delle donne a Roma ${ }^{19}$.

La Maraini raccoglie le testimonianze delle donne in lotta per i propri diritti, non solo alla Maddalena ma anche nelle sue precedenti attività nei quartieri della Magliana e di Centocelle. Tradurre in narrazione scenica le battaglie del movimento femminista, le permise di portare all'attenzione dei media tematiche scottanti come l'aborto, la prostituzione, l'omosessualità, lo sfruttamento del corpo della donna a fini commerciali. La sua scrittura diede voce e corpo a figure di donne marginalizzate dalla storia ufficiale, quali Suor Juana (1979), Maria Stuarda (1980), Veronica Franco, Camille Claudel, Isabella Andreini e la stessa Isabella di Morra, che dà il nome alla compagnia teatrale di Saviana Scalfi e Renata Zamengo per la quale Maraini scriverà i testi degli spettacoli dal 1977 al 1989 (Boggio, 2002; Peja, 2012).

Come racconta Piera Degli Esposti, il Teatro La Maddalena è stato per molte una casa, un fondamentale polo di attrazione, polivalente

19 Dacia Maraini fu una delle fondatrici insieme a Lù Leone, Francesca Pansa, Maricla Boggio, Edith Bruck, Giuliana Morandini, Annabella Ceriani, Rita Picchi e Saviana Scalfi. Il teatro ha permesso la creazione di un numero medio di 5-7 spettacoli annui (Maraini, 1979). Per il percorso personale della Maraini vedi Maraini e Murrali, 2013 e anche Peja, 2012. 
e molto frequentato, il luogo dove le esigenze delle attrici s'intrecciavano con quelle dello stesso movimento femminista:

Dunque si diceva "Vado alla Maddalena"...però, ecco, si sapeva che si poteva assistere a un corso di scrittura, di teatro che Dacia teneva, oppure si poteva andare a parlare, così, in qualche modo, ad avere uno scambio, un dibattito sul teatro, uno scambio... insomma si sapeva che Dacia sarebbe stata lì, era come una seconda volta in una seconda casa, dopo la Casa della donna di via del Governo Vecchio, la casa della Maddalena, un'altra diciamo casa, non dico casa del teatro, ma un proseguimento di quello che poi sarebbe diventato, io penso per più di un'attrice, per me sicuramente, un sostegno e una forza, questa forza femminile che riuscì a cambiare anche le fisionomie delle attrici, che non erano più queste bambolette, ma erano delle donne che erano anche delle guerriere, erano anche dei soldati, cioè delle donne che non erano più soltanto una propaggine dell'uomo. (Degli Esposti, 2013, pp. 94-95)

Bastano queste parole di Piera Degli Esposti per farci capire l'importanza per le teatranti di avere un posto dove esprimersi liberamente in un percorso di autorappresentazione libero dalla narrazione dominante nei teatri tradizionali, all'epoca tutti a conduzione maschile. Quel posto era autogestito e a direzione condivisa a tutti i livelli decisionali, dalla programmazione ai finanziamenti ${ }^{20}$ e vi fu possibile sperimentare la sorellanza, in altre parole assumere la relazione con le altre donne come punto di riferimento cui legare la costruzione della propria identità sia professionale che personale. Ma poteva anche capitare di usare il palcoscenico per sperimentare pratiche di Self-help ${ }^{21}$.

Proprio nello spazio del Teatro La Maddalena, le giovani "arrabbiate" di TheATRe diedero inizio nel 1979, secchi di vernice e pennellesse

20 La sede del Teatro della Maddalena a via della Stelletta (1973-1989) conteneva al piano superiore i locali della redazione della rivista femminista "effe". Per l'analisi dell'importanza di questa rivista rimando a Roberta Gandolfi, 2018 e 2019.

21 Conoscere il proprio corpo è obiettivo del Gruppo femminista per la medicina della donna che pratica il Self-help in Italia. L'uso dello speculum completo di uno specchio per esplorare l'interno della propria vagina porta ad accettare la diversità sessuale come valore e non come mancanza. Viene praticato sia nelle sedi dei collettivi che negli spazi del Teatro La Maddalena. 
in mano, alla trasformazione dello spazio scenico, ridipingendo le pareti di bianco: contestavano il teatro di testo in favore di nuove azioni performative. Fu questo il primo atto di una riappropriazione scenica che passava obbligatoriamente per la presa di possesso di un luogo dove fosse possibile sentirsi a proprio agio prima nelle prove e poi nello spettacolo, ma anche dopo, tra donne.

E allora i luoghi: spesso occupati o inaugurati da poco, culle dei nuovi linguaggi, spazi altri, rifugi che permettono di rigenerare il proprio immaginario, a volte coincidenti con gli spazi di riunione dei collettivi femministi, a volte rosicchiati all'avanguardia teatrale. Tra i primi la Casa Occupata dalle Donne a via del Governo Vecchio, enorme edificio in rovina, con grande cortile centrale e decine e decine di stanze su più piani, rifugio per ogni sorta d'iniziativa: dal centro contro la violenza alle donne alle assemblee animate che preparavano i cortei, dai gruppi di autocoscienza agli spettacoli comici. Qui, di giorno, si allenavano le ragazze del Teatro Viola che la sera si esibivano perlopiù a Zanzibar ${ }^{22}$, primo bar per sole donne a Roma dove ritrovarsi per ballare e divertirsi senza essere molestate dagli uomini.

I luoghi simbolo delle lotte, delle rivendicazioni e dei progetti del movimento femminista romano erano aperti solo alle donne.

Divennero nuovi luoghi performativi delle attrici anche le piazze delle manifestazioni, da ravvivare con parate e improvvisazioni a tema, e, almeno in parte, le "cantine" 23 : il Beat 72, l'Alberichino, il Teatro in Trastevere, tutti diretti da uomini tranne una, Spaziozero. Questo spazio, fondato da Manuela Morosini, nel 1979 ospita, per spettacoli e incontri di riflessione politico-culturale, Leo e Perla, Remondi e Caporossi, La Gaia Scienza, Cabaret Voltaire, Beat 72 (Morosini, 2014).

Luoghi del nuovo immaginario furono anche le gallerie d'arte, come l'Attico di via del Paradiso, aperta solo di notte da Fabio Sargen-

22 L'associazione culturale Zanzibar restò aperta dal 1978 al 1984 . Vedi il film: Zanzibar. Una storia d'amore, di Francesca Manieri e Monica Petrangeli, presentato al TekFestival di Roma nel 2009. Oggi quel luogo, passato di gestione, è uno delle centinaia di locali notturni di Trastevere.

23 Il fenomeno romano delle cantine usate come teatri, va considerato come quello di una specifica "scuola romana", cfr. bibliografia. 
tini, e altri spazi non convenzionali, inaugurati da Renato Nicolini per l'Estate Romana, tra questi l'Uccelliera di Villa Borghese e la piscina olimpica, scelti da Rossella Or per le sue performance.

\section{I REGISTRI ESPRESSIVI: INTERSEZIONI E RELAZIONI}

I registri espressivi delle teatranti romane di quel periodo si sono rivelati variegati e dissimili, a mano a mano che si sono susseguite le testimonianze. È questo il nostro più intenso e più difficile oggetto d'indagine, esplorare intersezioni e relazioni tra questi spettacoli e la ricerca teatrale dell'epoca, sospesa tra nuova drammaturgia e teatro immagine.

Se professioniste della scrittura come Dacia Maraini o Edith Bruck hanno imparato presto a distillare i materiali per i propri testi dalle storie vere che andavano incontrando nelle piazze e nei luoghi del movimento (aprendo così tutto un filone da esplorare che lega oralità e storie di vita, autocoscienza, teatro e società), altre hanno intrecciato l'espressione della soggettività femminile con sperimentazioni che venivano dalla scena del teatro di ricerca, della post-avanguardia, della performance art: l'uso di spazi nuovi, come la piscina di Mine-Ha; la mescolanza dei linguaggi, come in Shoe Show di Teatro Viola; l'utilizzo di un tappeto sonoro molto articolato che raddoppia le possibilità della parola, come nei monologhi di Rossella Or; gli elementi scenografici legati al teatro popolare e agit-prop, come l'uso delle grandi marionette di Deanna Frosini al Teatro La Maddalena o i pannelli dei cantastorie portati nelle periferie romane; lo straniamento nella recitazione, che permette di raccontare episodi scabrosi, come aborti e violenze alle donne, per immediatamente chiedere una presa di posizione da parte del pubblico, suscitare il dibattito, attivare le coscienze. Anche la querelle sull'uso del corpo in scena, contro o per la predominanza della parola sul corpo, permette di approfondire le varietà di percorsi di ricerca che si erano aperti in quegli anni (Giacobbe Borelli, 2018).

Il terreno dei registri espressivi è uno di quelli che andrebbe approfondito, a partire dalle fonti raccolte, continuando a tenere calde le domande che riguardano il rapporto tra femminismo e teatro: perché non c'è dubbio che il movimento femminista ha spinto drammaturgia 
e scrittura scenica a una nuova rappresentazione della donna in scena, che è andata strettamente intrecciandosi a un profondo cambiamento nella condizione professionale e personale delle attrici. E su questo tutto è ancora da scrivere...

Di una cosa siamo certe: l'incontro con il movimento delle donne è stato per tutte le attrici un incontro fondamentale con il proprio vissuto, con il proprio inconscio, un incontro con se stesse che ha preso caratteristiche formali diverse ma che ha visto sostanzialmente simile il bisogno urgente di esprimere istanze da troppo tempo inascoltate.

Ci congediamo aprendo una finestra sul capodanno del 1979, a casa dell'artista Mario Schifano, dove è ospite una cruda performance di TheATre: per terra, solo ferro, vetro, carta, metalli, materiali di scarto. Le performer si rotolano sul pavimento, pelle e vestiti lacerati, si lanciano contro le pareti fino a esaurirsi. "Per tagli e per ferite" l'intesa tra donne non dura. Non può durare. Il gruppo di TheATre, così come le ragazze di Teatro Viola, prenderà, già all'inizio degli anni Ottanta, altre strade, non teatrali. Ippolita Avalli pubblicherà nel 1982 per Feltrinelli il suo primo romanzo, Aspettando Ketty, un successo editoriale che racconta speranze e delusioni di una giovane artista degli anni Settanta.

\section{BIBLIOGRAFIA}

Bargiacchi, E.G., \& Sacchettini, R. (2017). Cento storie sul filo della memoria. Il "Nuovo Teatro" in Italia negli anni '70. Corazzano (Pi): Titivillus. Boggio, M. (Ed.). (2002). Le Isabelle. Dal teatro della Maddalena alla Isabella Andreini. Nardò: Besa.

Carandini, S. (Ed.). (2012). Memorie dalle cantine, Teatro di ricerca a Roma negli anni '60 e '70. Atti del Convegno (19-20 maggio 2008). Biblioteca Teatrale, 101-103. Roma: Bulzoni. Retrieved from https://bulzoni.it/it/ catalogo/n-101-103-gennaio-settembre-2012.html.

Centro di Documentazione del Movimento Femminista Romano (Ed.). (1976). Donnità. Cronache del movimento femminista romano. Roma: Editore Roma.

Degli Esposti, P. (2013). Testimonianze. Piera Degli Esposti. In D. Maraini, \& E. Murrali (Eds.), Il sogno del teatro, cronaca di una passione (pp. 94-95). Milano: BUR. 
De Marinis, M. (1987). 1947-1970. Milano: Bompiani.

Dolan, J. (1982). Teatro Viola's "Shoe-Show". The Drama Review, 26/3, 111-114 .

Fava, F. (2018). Donne di teatro a Roma ai tempi della mobilitazione femminista (1965-1985). In D. Orecchia \& L. Cavaglieri (Eds.), Fonti orali e teatro. Memoria, storia e performance (pp. 146-152). Bologna: Alma DL.

Fofi, G. (1974). Alcuni appunti sul romanzo. La Storia. Ombre rosse, 7, n. s., 89-98.

Gandolfi, R. (2018). "effe" (1972-1983) e il discorso femminista sulla corporeità femminile: il cinema e l'industria culturale. Smarginature, Arabeschi, 41. Retrieved from http://www.arabeschi.it/41-effe-1972-1983-e-il-dis corso-femminista-sulla-corporeit--femminile-cinema-lindustria-culturale/.

Gandolfi, R. (2019, to appear). Teatro e danza su "effe" (1973-1982): la rivista come archivio del discorso femminista sul corpo. Itinera, 18.

Giacobbe Borelli, M. (2018). La querelle del nudo sulle scene dei teatri d'avanguardia a Roma negli anni Sessanta e Settanta. Smarginature, Arabeschi, 45. Retrieved from http://www.arabeschi.it/45-la-querelle-del-nudo-sul le-scene-dei-teatri-davanguardia-a-roma-negli-anni-sessanta-e-settanta.

Lussana, F. (2012). Il movimento femminista in Italia (esperienze, storie, memorie). Roma: Carocci.

Maraini, D. (1976). Strappiamo il sipario, facciamone dei vestiti. effe, 7-8, 24-26.

Maraini, D., \& Collettivo La Maddalena (1979, November 28). Vogliamo fare teatro da barricata. Quotidiano Donna, p. 12.

Maraini, D. (2001). Dialogo di una prostituta con un suo cliente. Milano: BUR.

Maraini, D., \& Murrali, E. (Eds.). (2013). Il sogno del teatro, cronaca di una passione. Milano: BUR.

Margiotta, S. (2013). Il Nuovo Teatro in Italia 1968-1975. Corazzano (Pi): Titivillus.

Morante, E. (1974). La storia, Torino: Einaudi.

Orecchia, D., \& Cavaglieri, L. (Eds.). (2018). Fonti orali e teatro. Memoria storia e performance. Bologna: Alma DL.

Peja, L. (2012). La drammaturgia di Dacia Maraini. Paradossi di un teatro di militanza e poesia. Comunicazioni Sociali. Journal of Media, Performing Arts and Cultural Studies, 1, 115-135.

Stelliferi, P. (2015). Il femminismo a Roma negli anni Settanta: percorsi esperienze e memorie dei collettivi di quartiere. Bologna: Bonomia University Press. 
Tafuri, C., \& Baronio, D. (Eds.). (2018). Ivrea cinquanta. Mezzo secolo di Nuovo Teatro in Italia 1967-2017. Atti del Convegno (Genova, 5-7 maggio 2017). Genova: Akropolis Libri.

Valentino, M. (2015). Il Nuovo Teatro in Italia 1976-1985. Corazzano (Pi): Titivillus.

Valentini, V. (2015). Nuovo Teatro Made in Italy 1963-2013. Roma: Bulzoni.

Visone, D. (2010). La nascita del Nuovo Teatro in Italia 1959-1967. Corazzano (Pi): Titivillus.

Interviste audio del Progetto ORMETE, ancora inedite e in parte disponibili al sito:

http://patrimoniorale.ormete.net/progetto/donne-di-teatro-a-roma-aitempi-della-mobilitazione-femminista-1965-1985

Bassignano I., (2016, 1 settembre). Progetto ORMETE, intervista a Ida Bassignano di Francesca Fava e Roberta Gandolfi, Roma.

Bramini S., (2015, 20 gennaio). Progetto ORMETE, intervista a Sista Bramini di Francesca Fava. Roma.

Morosini M., (2014, 10 dicembre). Progetto ORMETE, intervista a Manuela Morosini di Francesca Fava. Roma.

Or R., (2015, 2 luglio). Progetto ORMETE, intervista a Rossella Or di Francesca Fava e Maia Giacobbe Borelli. Roma.

Poli L., (2014, 3 luglio). Progetto ORMETE, intervista a Lucia Poli di Francesca Fava. Roma.

Prati M., (2015, 13 luglio). Progetto ORMETE, intervista a Marilù Prati di Maia Giacobbe Borelli e Francesca Fava. Roma.

\footnotetext{
Riassunto: L'intervento, che attinge a una ricerca in corso nel quadro di ORMETE (Oralità Memoria Teatro: www.ormete.net) Donne di teatro a Roma ai tempi della mobilitazione femminista (1965-1985), cerca di mettere a fuoco gli intrecci fra mobilitazioni femministe e protagonismo delle attrici a Roma, fra il 1974 e il 1979. L'affermazione di un'identità femminile forte e anticonformista caratterizza il racconto di tutte queste donne, determinate a trovare la strada per esprimere il proprio immaginario e la propria diversa presenza nel mondo romano della ricerca teatrale. Molte si sono associate in compagnie o collettivi, alcune hanno tentato la strada della drammaturgia, altre hanno scelto la forma della performance più trasgressiva. L'indagine mette l'accento sui molti luoghi di Roma dove i diversi linguaggi scenici hanno trovato la luce.
}

Parole chiave: avanguardia teatrale, femminismo, Nuovo Teatro, Roma, 1974-1979 